\title{
Prenatal Risk Factors for PHACE Syndrome: a Study using the PHACE Syndrome International Clinical Registry and Genetic Repository
}

\author{
Joy Wan, M.D. ${ }^{1}$, Jack Steiner, B.S. ${ }^{2}$, Eulalia Baselga, M.D. ${ }^{3}$, Francine Blei, M.D. ${ }^{4}$, Maria \\ Cordisco, M.D. ${ }^{5}$, Maria C. Garzon, M.D. ${ }^{6}$, Deborah S. Goddard, M.D. ${ }^{7}$, Anita Haggstrom, M.D. \\ ${ }^{8}$, Alfons Krol, M.D. ${ }^{9}$, Ilona J. Frieden, M.D. ${ }^{10}$, Denise Metry, M.D. ${ }^{11}$, Kimberly D. Morel, M.D. \\ 6 , Judith M.A. Verhagen, M.D., Ph.D. ${ }^{12}$, Orli Wargon, M.D. ${ }^{13}$, Beth A. Drolet, M.D. ${ }^{14}$, and \\ Dawn H. Siegel, M.D. ${ }^{14}$
}

${ }^{1}$ Department of Dermatology, University of Pennsylvania School of Medicine, Philadelphia, PA ${ }^{2}$ Department of Dermatology, Medical College of Wisconsin, Milwaukee, WI ${ }^{3} \mathrm{Hospital}$ de la Santa Creu i Sant Pau, Barcelona, Spain ${ }^{4}$ Vascular Anomalies Program, Lenox Hill Hospital, Northwell Health, New York, NY ${ }^{5}$ Department of Dermatology, University of Rochester Medical Center, Rochester, NY ${ }^{6}$ Departments of Dermatology and Pediatrics, Columbia University, New York, NY ${ }^{7}$ Kuchnir Dermatology \& Dermatologic Surgery, Framingham and Milford, MA ${ }^{8}$ Department of Dermatology, School of Medicine, Indiana University, Indianapolis, IN ${ }^{9}$ Departments of Dermatology and Pediatrics, Oregon Health \& Science University, Portland, OR ${ }^{10}$ Department of Dermatology, University of California San Francisco, San Francisco, CA ${ }^{11}$ Department of Dermatology, Texas Children's Baylor, Houston, TX ${ }^{12}$ Department of Clinical Genetics, Erasmus University Medical Center, Rotterdam, The Netherlands ${ }^{13}$ Department of Pediatric Dermatology, Sydney Children's Hospital School of Women's and Children's Health, University of New South Wales, Sydney, New South Wales, Australia ${ }^{14}$ Departments of Dermatology and Pediatrics, Section of Pediatric Dermatology, Medical College of Wisconsin, Milwaukee, WI

\section{Abstract \\ The cause of PHACE syndrome is unknown. In a study of 218 patients, we examined potential prenatal risk factors for PHACE syndrome. Rates of pre-eclampsia and placenta previa in affected individuals were significantly higher than in the general population. No significant risk factor differences were detected between males and females.}

\section{Keywords \\ disease pathogenesis; hemangioma; perinatal risk factors}

Corresponding author: Joy Wan, M.D., Hospital of the University of Pennsylvania, Department of Dermatology, 3600 Spruce Street, 2 East Gates Building, Philadelphia, PA 19104. Tel: 215-614-1620; Fax: 215-615-3140; Joy.Wan@uphs.upenn.edu.

Publisher's Disclaimer: This is a PDF file of an unedited manuscript that has been accepted for publication. As a service to our customers we are providing this early version of the manuscript. The manuscript will undergo copyediting, typesetting, and review of the resulting proof before it is published in its final citable form. Please note that during the production process errors may be discovered which could affect the content, and all legal disclaimers that apply to the journal pertain.

The authors declare no conflicts of interest. 
PHACE syndrome (OMIM 606519) is a disorder characterized by Posterior fossa brain malformations, large or segmental Hemangiomas, Arterial cerebrovascular and/or Cardiovascular anomalies, Eye abnormalities, and ventral developmental defects. The cause of PHACE syndrome is unknown, and while genetic factors have been proposed, prenatal risk factors for PHACE syndrome have not been thoroughly examined (1-3). Risk factors for infantile hemangiomas have been previously described, including female sex, older maternal age, prematurity, low birth weight, and multiple gestation. Published reports of patients with PHACE syndrome have found that, unlike patients with infantile hemangiomas alone, those with PHACE syndrome are more likely to be female, are generally born full term, and are more often the result of single gestation pregnancies $(4,5)$. However, previous studies describing prenatal factors in PHACE syndrome were based on fewer than 35 patients each $(5,6)$. Thus, we sought to update the existing literature by using a large, international clinical registry to examine potential prenatal risk factors for PHACE syndrome.

\section{Methods}

We performed a cross-sectional analysis of patients enrolled in the PHACE Syndrome International Clinical Registry and Genetic Repository through August 2016. (7-9). Patients were recruited for enrollment into the registry via in-person visits at the Children's Hospital of Wisconsin, referrals from the PHACE Syndrome Community advocacy group, direct enrollment via the PHACE syndrome registry website, and direct referrals from other physicians and genetic counselors to the registry investigators. Only patients with a confirmed clinical diagnosis of PHACE syndrome after review of medical records were included in the analysis. At the time of registry enrollment, the patient or his or her caregiver completed a questionnaire assessing patient demographics, prenatal characteristics, and maternal pregnancy history. Additional questions were added to the registry enrollment questionnaire over time, including those on maternal medication use, infection, trauma, and near miscarriage during the pregnancy; thus, only a subset of registry participants completed these particular questions. Descriptive statistics were performed, and the 1-sample test of proportion was used to compare our data to prevalence rates in the general population as obtained from published literature or US national health statistics. An exploratory analysis comparing male and female patients with respect to prenatal characteristics was also performed; the Fisher exact test was used to compare categorical variables, and the Student t-test or Wilcoxon rank-sum test was used to compare continuous variables as warranted by the distribution of the data. All patients provided informed consent at the time of registry enrollment, and the study was approved by the Institutional Review Board at the Children's Hospital of Wisconsin.

\section{Results}

As of August 2016, a total of 218 patients had been enrolled in the PHACE Syndrome International Clinical Registry and Genetic Repository. Of the patients with completed responses on the enrollment questionnaire, 176 out of 218 (80.7\%) were female and 170 out of $196(86.7 \%)$ were white (Table 1). Of the 170 patients who provided ethnicity data, 42 
(24.7\%) were Hispanic or Latino. The median birth weight of patients was 3317 (interquartile range (IQR) 2948-3700) grams, with the majority of patients being of normal weight (Table 1). Low birth weight and very low birth weight were reported in $7.4 \%$ and $1.1 \%$, respectively, of the patients and are similar to the U.S. population prevalence rates of $8.0 \%(\mathrm{p}=0.76)$ and $1.4 \%(\mathrm{p}=0.69)$, respectively (10). The median gestational age at birth was 39 (IQR 38-40) weeks, with $90.9 \%$ of patients born at full term (Table 1). The mean maternal age was 30.6 (standard deviation [SD] 5.91) years. The birth month of PHACE syndrome patients did not appear to have any apparent seasonal trends (Table 1).

Of the 218 patients in the registry, 193 patients were asked about prenatal procedures, prenatal complications such as pre-eclampsia and placental abnormalities, and maternal pregnancy history on the enrollment questionnaire. The median number of total pregnancies in the mothers of PHACE syndrome patients was 2 (IQR 2-3). Of respondents, $66.1 \%$ of mothers had no history of miscarriages, $23.6 \%$ had one prior miscarriage, and $10.3 \%$ reported 2 or more previous miscarriages (Table 2). Among the 41 mothers with a history of 1 miscarriage, 16 mothers reported the trimester in which the miscarriage occurred: 13 $(81.3 \%)$ in first trimester, $2(12.5 \%)$ in second trimester, and $1(6.3 \%)$ in third trimester (Table 2). Among the 18 mothers in our cohort with a history of 2 or more miscarriages, there were 46 miscarriages in total; of these, 21 miscarriages had associated data on trimester of occurrence: 18 (85.7\%) in first trimester, 3 (14.3\%) in second trimester, and none in third trimester (Table 2). Three mothers reported associated fetal anomalies with their miscarriages: Turner syndrome, trisomy 19, and anencephaly. Twenty-two (11.7\%) mothers indicated a history of maternal infertility, which was similar to the US population prevalence of infertility of $12 \%(\mathrm{p}=0.90)(11,12)$. Twenty-three $(12.3 \%)$ patients were conceived using fertility therapy, with in vitro fertilization being the most commonly used type of treatment (Table 2). Eleven (5.9\%) patients were the product of multiple gestation pregnancies, a rate that is slightly higher but not significantly different from the U.S. population prevalence of multiple gestation of $3.5 \%(\mathrm{p}=0.08)(13)$. Amniocentesis and chorionic villus sampling were performed in $9.5 \%$ and $2.2 \%$ of the pregnancies, respectively (Table 2). A total of $16(8.5 \%)$ pregnancies were reported to be complicated by preeclampsia, which is significantly higher than the population prevalence rate of $3.4 \%$ $(\mathrm{p}<0.001)$ (14). Of the 16 patients with pre-eclampsia, 10 (62.5\%) were diagnosed in the third trimester, $1(6.3 \%)$ in the second trimester, none in the first trimester, and $5(31.3 \%)$ were unknown. Ten patients reported the specific gestational week of pre-eclampsia diagnosis, with a median age of 35.3 (IQR 33-38, range 21-39) weeks. Placental abnormality was reported in $21(11.2 \%)$ pregnancies, with placenta previa being the most commonly reported abnormality (Table 2). The rate of placenta previa within our cohort was $3.2 \%$, which is significantly higher than the North American population prevalence of $0.29 \%$ $(\mathrm{p}<0.001)(15)$. Of the 6 patients with placenta previa, $2(33.3 \%)$ were diagnosed in the first trimester, $1(16.7 \%)$ in the second trimester, $1(16.7 \%)$ in the third trimester, and $2(33.3 \%)$ were unknown. Fourteen (7.4\%) pregnancies were complicated by maternal diabetes (Table 2).

The 50 most recently enrolled participants in the registry were also asked questions about prescription medication use, infection, trauma, near miscarriage, and bleeding during pregnancy on their enrollment survey. Of 47 participants with completed responses, 16 
(34.0\%) mothers had used prescription medications during their pregnancy, with acetaminophen and anti-emetics being the most commonly reported medications (Table 2). Prenatal infection was reported by $6(12.8 \%)$ mothers, and prenatal trauma was reported by $4(8.5 \%)$ mothers (Table 2$)$. Only 1 mother had a near miscarriage during her pregnancy, and $5(10.9 \%)$ mothers reported bleeding during the pregnancy (Table 2). There was no overlap between the mothers reporting trauma and those reporting bleeding.

Given the female predominance in PHACE syndrome, we examined whether female and male patients with PHACE syndrome differed with respect to their prenatal characteristics and maternal pregnancy history. We observed no significant differences between males and females, including with respect to birth weight, gestational age, and rates of multiple gestation, pre-eclampsia, and placental abnormalities (data not shown). Maternal characteristics such as age, number of pregnancies and miscarriages, history of infertility, and use of fertility treatment also did not differ between the 2 groups (data not shown).

\section{Discussion}

In this study, we describe the prenatal characteristics of a large cohort of patients with PHACE syndrome. We observed a female-to-male ratio of 4.2, which is lower than the female-to-male ratio of 7.3 that was previously described in smaller cohorts of PHACE syndrome patients but remains higher than the female-to-male ratio of 2.4 observed in a large prospective study of isolated infantile hemangiomas $(4,5)$. Although one previous study compared disease severity and presentation between male and female patients with PHACE syndrome and found no major differences with the exception of more structural brain anomalies in males, no study to our knowledge has compared prenatal characteristics between males and females with PHACE syndrome. In our exploratory analysis, we found no significant differences between males and females with respect to potential prenatal risk factors.

Similar to previous reports of PHACE syndrome patients, we found that the majority of patients in our registry are of white race, normal birth weight, and full term gestation $(5,6)$. It is interesting that although sporadic infantile hemangiomas are often associated with prematurity, PHACE syndrome does not appear to be, the reasons for which remain unknown. In the case of sporadic infantile hemangiomas, it has been postulated that placental complications in utero drive both hemangioma development and prematurity (16). If such placental abnormalities also drove hemangioma development in PHACE syndrome, it is unclear why such placental abnormalities would not also lead to prematurity in PHACE syndrome as they are thought to do in the case of infantile hemangiomas. Perhaps there is a compensatory mechanism or a genetic or epigenetic alteration present in PHACE syndrome but absent from sporadic infantile hemangiomas that prevents the placental abnormalities from inducing premature labor. However, this is speculative, and further research is needed to elucidate the mechanisms underlying these differences between PHACE syndrome and sporadic infantile hemangiomas.

We observed significantly higher reported rates of pre-eclampsia and placental abnormalities, specifically placenta previa, in our cohort of PHACE syndrome patients than 
in the general population, suggesting that these complications may be potential risk factors for PHACE syndrome. In reviewing the pregnancies with pre-eclampsia or placental abnormalities, we did not see any striking features unique to these pregnancies. Four of the 16 patients with pre-eclampsia had been asked about medications, infections, trauma, near miscarriage, and bleeding during pregnancy. One of the 4 patients reported prescription medication use (levothyroxine), although none reported infection, trauma, near miscarriage, or bleeding. Among the 21 patients reporting placental anomalies, 8 had been asked about medications, infections, trauma, near miscarriage, and bleeding during pregnancy but only 6 patients provided responses. Of these 6 patients, 1 reported prescription medication use (ondansetron), 4 reported no medication use, and 1 was unknown. Infection was reported by 1 of the 6 patients (bacterial vaginosis and yeast infection), and trauma was reported by 1 of the patients (a fall). No patients reported near miscarriage, and 1 patient reported bleeding due to her low-lying placenta. We also further examined the structural brain and vascular anomalies seen in this population. Of the 16 patients with pre-eclampsia, data on structural brain and vascular abnormalities were available for 15 patients: $9(60.0 \%)$ had brain or posterior fossa abnormalities; $12(80.0 \%)$ had arterial anomalies; and $12(80.0 \%)$ had cardiac abnormalities. Of the 21 patients with placental abnormalities, we had data on structural abnormalities for 20 patients: $8(40.0 \%)$ had brain or posterior fossa abnormalities; $14(70.0 \%)$ had arterial anomalies; and $11(55.0 \%)$ had cardiac abnormalities. Although these sample sizes are small, the frequencies of specific anomalies are generally similar to what we see across the entire cohort: $41 \%$ had brain or posterior fossa abnormalities, $80 \%$ had arterial anomalies, and 54\% had cardiac abnormalities. It is thus difficult to draw any conclusions from our data about whether pre-eclampsia or placental abnormalities have a direct impact on the structural abnormalities. Moreover, rates of pre-eclampsia and placenta previa are also similarly elevated among patients with infantile hemangiomas in general. In a study by Haggstrom et al, in over 1000 children with infantile hemangiomas, the rates of placenta previa and pre-eclampsia were $3.3 \%$ and $11.8 \%$, respectively (4), which are similar to the rates observed in our study. In another smaller study of 594 infants, placental abnormalities were noted in $37 \%$ of infants with infantile hemangiomas but only in $18 \%$ of those without hemangiomas (17). These findings suggest that placental abnormalities and pre-eclampsia may not be risk factors specific to PHACE syndrome but are instead risk factors for the hemangioma component of PHACE syndrome.

Several mechanisms have been posited to explain the association of placental abnormalities and pre-eclampsia with hemangiomas. As infantile hemangiomas share histochemical features and a similar programmed life cycle with placental tissue, it has been suggested that hemangiomas may be derived from placental tissue $(18,19)$. Pre-eclampsia and placenta previa can be associated with placental insufficiency resulting in hypoxia. Hypoxia has been proposed to be an important driver of hemangioma development and therefore may be a risk factor in PHACE syndrome as well $(20,21)$.

Although infantile hemangiomas have been associated with the use of in vitro fertilization in previous studies (22-24), the rates of maternal infertility and fertility treatment in our study were not significantly different from population prevalence rates, suggesting that these are less likely risk factors for PHACE syndrome. However, $34 \%$ of mothers in the registry reported a history of at least 1 miscarriage and this rate is significantly higher than 
population estimates of miscarriage of $20 \%$ (12). Finally, we did not observe in our cohort any major patterns in the season of birth or rates of amniocentesis, chorionic villus sampling, maternal diabetes, prenatal trauma and infection, or prescription medication use.

Our study has limitations. First, non-response to certain items in the enrollment questionnaire led to over $10 \%$ missing data on several variables including birth weight, gestational age, and maternal age. However, the amount of unreported data on pregnancy history and prenatal characteristics was generally low, and as the study is primarily descriptive, we did not perform multiple imputation. Second, our study relied on patient selfreport, which may be subject to limitations in recall. As pre-eclampsia and placental anomalies were self-reported by the mothers in our study, the ascertainment of maternal conditions may differ from that used in the studies from which population prevalence rates were derived. Third, we did not have a comparator group of patients without PHACE syndrome to directly compare with our cohort, but we instead used population prevalence rates from the literature. Fourth, we may have been underpowered to detect statistically significant differences between males and females in our exploratory analysis, thus limiting our ability to draw definitive conclusions from those findings. Finally, the number of patients who were surveyed about prenatal medications, infection, trauma, and bleeding was limited in sample size to only the most recently enrolled 50 patients.

Our study supports previous findings that patients with PHACE syndrome are mostly female and white but less likely to be premature or of low birth weight than patients with isolated infantile hemangiomas. Our results also suggest that pre-eclampsia and placenta previa may be potential risk factors for PHACE syndrome, but further work is needed to distinguish their impact on the development of infantile hemangiomas alone versus PHACE syndrome specifically. Overall, no single prenatal risk factor appears to drive PHACE syndrome, and to date, no clear genetic cause has been identified. As our understanding of the genetics of PHACE syndrome grows, it will also be imperative to investigate the interaction between prenatal risk factors and genetic influences in the pathogenesis of PHACE syndrome.

\section{Acknowledgments}

Supported by the National Institutes of Health (T32-AR007465 [to J.W.] and R01-AR064258 [to D.S.]). We also thank the Society for Pediatric Dermatology, Dermatology Foundation, Greater Milwaukee Foundation, and NOVA for prior funding that supported the PHACE Syndrome International Clinical Registry and Genetic Repository.

We are grateful to the PHACE Syndrome Community, PHACE Foundation Canada, Shawna Brown (Department of Dermatology, Medical College of Wisconsin), Angela Beltrame (Section of Pediatric Dermatology, Medical College of Wisconsin), and Katherine Mueller (Department of Dermatology, Medical College of Wisconsin) for their support of and contributions to this work.

\section{Abbreviations \\ IQR interquartile range \\ SD standard deviation}




\section{References}

1. Sullivan CT, Christian SL, Shieh JT, Metry D, Blei F, Krol A, et al. X chromosome-inactivation patterns in 31 individuals with PHACE syndrome. Mol Syndromol. 2013 Mar.4:114-8. [PubMed: 23653582]

2. Siegel DH, Shieh JT, Kwon EK, Baselga E, Blei F, Cordisco M, et al. Copy number variation analysis in 98 individuals with PHACE syndrome. J Invest Dermatol. 2013 Mar.133:677-84. [PubMed: 23096700]

3. Mitchell S, Siegel DH, Shieh JT, Stevenson DA, Grimmer JF, Lewis T, et al. Candidate locus analysis for PHACE syndrome. Am J Med Genet A. 2012 Jun.158A:1363-7. [PubMed: 22544659]

4. Haggstrom AN, Drolet BA, Baselga E, Chamlin SL, Garzon MC, et al. Hemangioma Investigator Group. Prospective study of infantile hemangiomas: Demographic, prenatal, and perinatal characteristics. J Pediatr. 2007 Mar.150:291-4. [PubMed: 17307549]

5. Metry DW, Haggstrom AN, Drolet BA, Baselga E, Chamlin S, Garzon M, et al. A prospective study of PHACE syndrome in infantile hemangiomas: Demographic features, clinical findings, and complications. Am J Med Genet A. 2006 May 1.140:975-86. [PubMed: 16575892]

6. Haggstrom AN, Garzon MC, Baselga E, Chamlin SL, Frieden IJ, Holland K, et al. Risk for PHACE syndrome in infants with large facial hemangiomas. Pediatrics. 2010 Aug.126:e418-26. [PubMed: 20643720]

7. Bayer ML, Frommelt PC, Blei F, Breur JM, Cordisco MR, Frieden IJ, et al. Congenital cardiac, aortic arch, and vascular bed anomalies in PHACE syndrome (from the international PHACE syndrome registry). Am J Cardiol. 2013 Dec 15.112:1948-52. [PubMed: 24079520]

8. Brosig CL, Siegel DH, Haggstrom AN, Frieden IJ, Drolet BA. Neurodevelopmental outcomes in children with PHACE syndrome. Pediatr Dermatol. 2016 Jul.33:415-23. [PubMed: 27291925]

9. Yu J, Siegel DH, Drolet BA, Blei F, Epstein LG, Metry D, et al. Prevalence and clinical characteristics of headaches in PHACE syndrome. J Child Neurol. 2016 Mar.31:468-73. [PubMed: 26271792]

10. National center for health statistics. Atlanta, GA: Centers for Disease Control and Prevention; 2016 Jul 6. Birthweight and gestation [homepage on the Internet]. 2016 Available from: https:// www.cdc.gov/nchs/fastats/birthweight.htm [cited October 10, 2016]

11. Reproductive health. Atlanta, GA: Centers for Disease Control and Prevention; 2016 Apr 14. Infertility FAQs [homepage on the Internet]. 2016 Available from: http://www.cdc.gov/ reproductivehealth/Infertility/index.htm [cited October 10, 2016]

12. Kim ME, Cancel M, Metry D, Strawn EY, Drolet BA, Chiu YE, et al. Evaluation of maternal history of miscarriage, infertility, and in-vitro fertilization use as associated factors in PHACE. Br J Dermatol. 2017 Jan 27.doi: 10.1111/bjd.15341

13. National center for health statistics. Atlanta, GA: Centers for Disease Control and Prevention; 2016 Jun 13. Multiple births [homepage on the Internet]. 2016 Available from: http://www.cdc.gov/ nchs/fastats/multiple.htm [cited October 10, 2016]

14. Ananth CV, Keyes KM, Wapner RJ. Pre-eclampsia rates in the United States, 1980-2010: Ageperiod-cohort analysis. BMJ. 2013 Nov 7.347:f6564. [PubMed: 24201165]

15. Cresswell JA, Ronsmans C, Calvert C, Filippi V. Prevalence of placenta praevia by world region: A systematic review and meta-analysis. Trop Med Int Health. 2013 Jun.18:712-24. [PubMed: 23551357]

16. Boye E, Jinnin M, Olsen BR. Infantile hemangioma: Challenges, new insights, and therapeutic promise. J Craniofac Surg. 2009 Mar; 20(Suppl 1):678-84. [PubMed: 19190505]

17. Munden A, Butschek R, Tom WL, Marshall JS, Poeltler DM, Krohne SE, et al. Prospective study of infantile haemangiomas: Incidence, clinical characteristics and association with placental anomalies. Br J Dermatol. 2014 Apr.170:907-13. [PubMed: 24641194]

18. Ritter MR, Butschek RA, Friedlander M, Friedlander SF. Pathogenesis of infantile haemangioma: New molecular and cellular insights. Expert Rev Mol Med. 2007 Nov 29.9:1-19.

19. North PE, Waner M, Mizeracki A, Mrak RE, Nicholas R, Kincannon J, et al. A unique microvascular phenotype shared by juvenile hemangiomas and human placenta. Arch Dermatol. 2001 May.137:559-70. [PubMed: 11346333] 
20. Colonna V, Resta L, Napoli A, Bonifazi E. Placental hypoxia and neonatal haemangioma: Clinical and histological observations. Br J Dermatol. 2010 Jan.162:208-9. [PubMed: 19863512]

21. Drolet BA, Frieden IJ. Characteristics of infantile hemangiomas as clues to pathogenesis: Does hypoxia connect the dots? Arch Dermatol. 2010 Nov.146:1295-9. [PubMed: 21079070]

22. Dickison P, Christou E, Wargon O. A prospective study of infantile hemangiomas with a focus on incidence and risk factors. Pediatr Dermatol. 2011 Nov-Dec;28:663-9. [PubMed: 21995808]

23. Chen XD, Ma G, Chen H, Ye XX, Jin YB, Lin XX. Maternal and perinatal risk factors for infantile hemangioma: A case-control study. Pediatr Dermatol. 2013 Jul-Aug;30:457-61. [PubMed: 23278441]

24. Blei F, McElhinney DB, Guarini A, Presti S. Cardiac screening in infants with infantile hemangiomas before propranolol treatment. Pediatr Dermatol. 2014 Jul-Aug;31:465-70. [PubMed: 24889812] 


\section{Table 1}

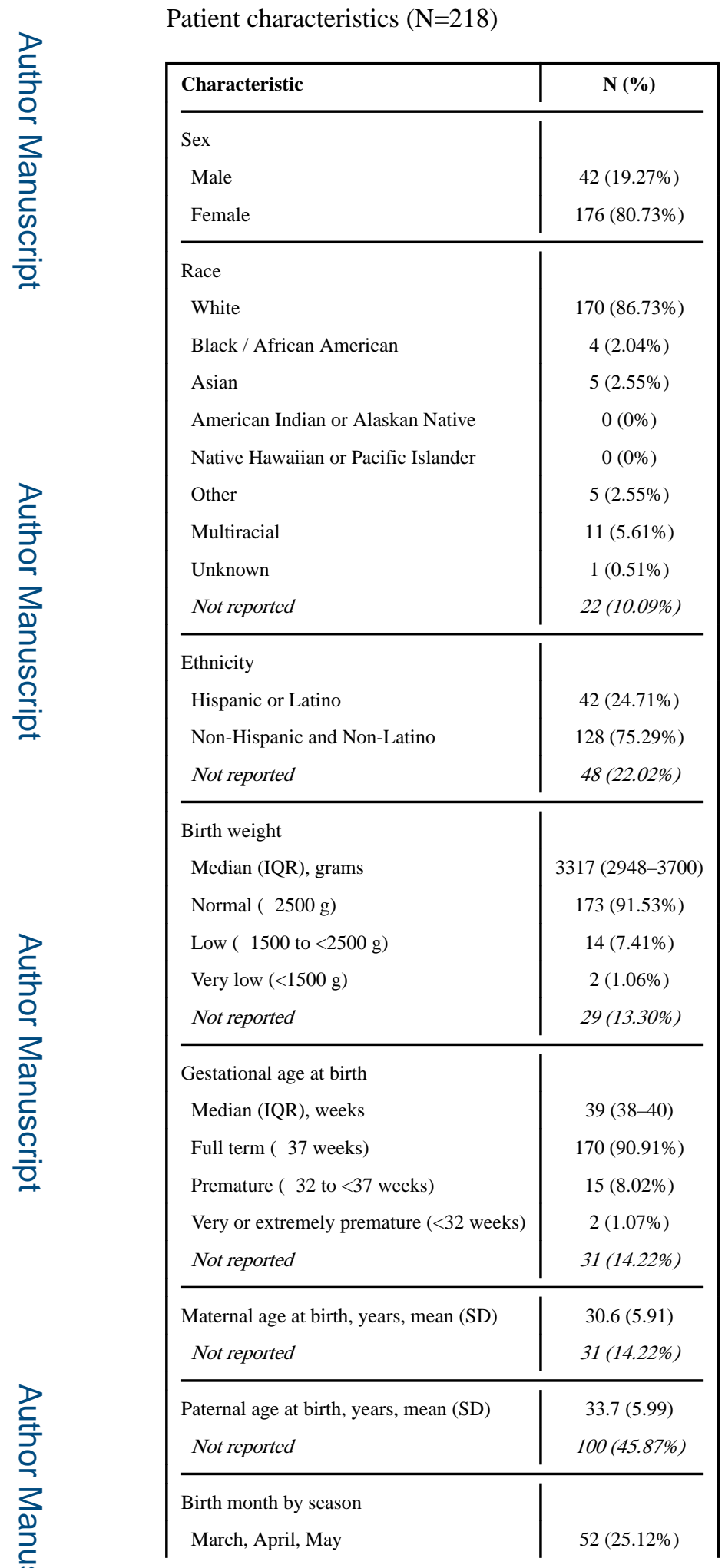




\begin{tabular}{|l|c|}
\hline Characteristic & N (\%) \\
\hline June, July, August & $68(32.85 \%)$ \\
September, October, November & $47(22.71 \%)$ \\
December, January, February & $40(19.32 \%)$ \\
Not reported & $11(5.05 \%)$ \\
\hline
\end{tabular}

$\mathrm{IQR}$, interquartile range; SD, standard deviation 
Table 2

Maternal pregnancy history and prenatal characteristics of patients

\begin{tabular}{|c|c|}
\hline Characteristic & $\mathbf{N}(\%)$ \\
\hline \multicolumn{2}{|l|}{ Total $N=193$} \\
\hline Total number of pregnancies, median (IQR) & $2.0(2.0-3.0)$ \\
\hline Not reported & $12(6.22 \%)$ \\
\hline Total number of miscarriages, median (IQR) & $0(0-1)$ \\
\hline 0 & $115(66.09 \%)$ \\
\hline 1 & $41(23.56 \%)$ \\
\hline 2 & $13(7.47 \%)$ \\
\hline 3 & $2(1.15 \%)$ \\
\hline 4 & $1(0.57 \%)$ \\
\hline 5 & $2(1.15 \%)$ \\
\hline Not reported & $19(9.84 \%)$ \\
\hline \multicolumn{2}{|l|}{ Trimester of reported miscarriages, if any } \\
\hline \multicolumn{2}{|l|}{ Among those with 1 miscarriage ( $\mathrm{N}=41$ miscarriages) } \\
\hline $1^{\text {st }}$ trimester & $13(81.25 \%)$ \\
\hline $2^{\text {nd }}$ trimester & $2(12.50 \%)$ \\
\hline $3^{\text {rd }}$ trimester & $1(6.25 \%)$ \\
\hline Not reported & $25(60.98 \%)$ \\
\hline \multicolumn{2}{|l|}{ Among those with $\geq 2$ miscarriages ( $N=46$ miscarriages) } \\
\hline $1^{\text {st }}$ trimester & $18(85.71 \%)$ \\
\hline $2^{\text {nd }}$ trimester & $3(14.29 \%)$ \\
\hline $3^{\text {rd }}$ trimester & $0(0 \%)$ \\
\hline Not reported & $25(54.35 \%)$ \\
\hline \multicolumn{2}{|l|}{ History of maternal infertility } \\
\hline Yes & $22(11.70 \%)$ \\
\hline No & $164(87.23 \%)$ \\
\hline Unknown & $2(1.06 \%)$ \\
\hline Not reported & $5(2.59 \%)$ \\
\hline \multicolumn{2}{|l|}{ Fertility treatment } \\
\hline Yes & $23(12.30 \%)$ \\
\hline No & $162(86.63 \%)$ \\
\hline Unknown & $2(1.07 \%)$ \\
\hline Not reported & $6(3.11 \%)$ \\
\hline \multicolumn{2}{|l|}{ If yes, type(s) of fertility treatment } \\
\hline In vitro fertilization & $14(60.87 \%)$ \\
\hline Clomiphene & $5(21.74 \%)$ \\
\hline Artificial insemination & $5(21.74 \%)$ \\
\hline
\end{tabular}




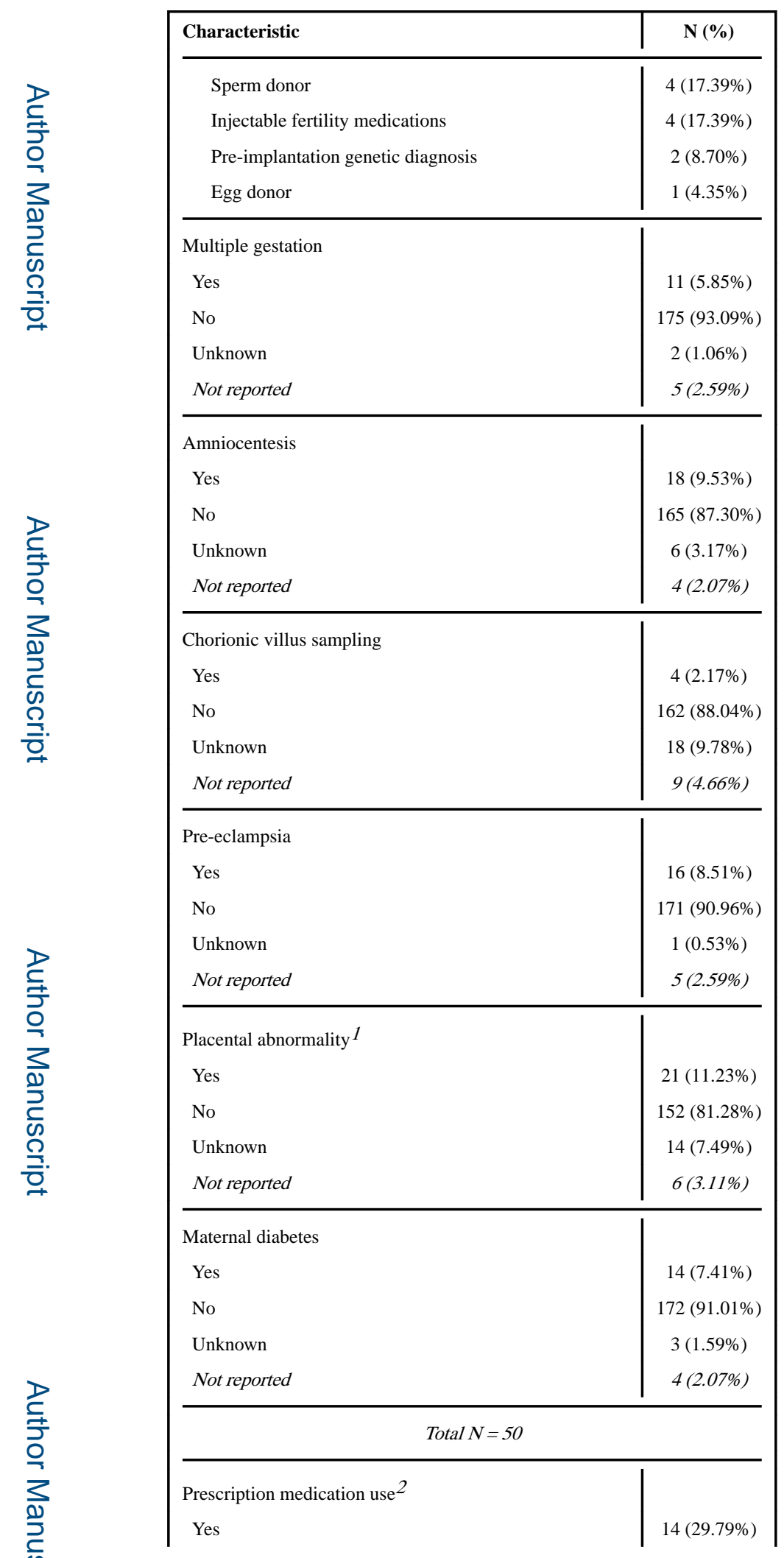




\begin{tabular}{|l|c|}
\hline Characteristic & $\mathbf{N}(\%)$ \\
\hline No & $31(65.96 \%)$ \\
Unknown & $2(4.26 \%)$ \\
Not reported & $3(6.00 \%)$ \\
\hline Infection ${ }^{3}$ & \\
Yes & $6(12.77 \%)$ \\
No & $41(87.23 \%)$ \\
Not reported & $3(6.00 \%)$ \\
\hline Trauma or accident ${ }^{4}$ & \\
Yes & $4(8.51 \%)$ \\
No & $43(91.49 \%)$ \\
Not reported & $3(6.00 \%)$ \\
\hline Near miscarriage & $1(2.27 \%)$ \\
Yes & $43(97.73 \%)$ \\
No & $6(12.00 \%)$ \\
Not reported & $5(10.87 \%)$ \\
\hline Bleeding during pregnancy & $4(89.13 \%)$ \\
Yes & \\
No & \\
Not reported & \\
\hline
\end{tabular}

$\mathrm{IQR}$, interquartile range

${ }^{1}$ Placental abnormalities: placenta previa (6 patients), two vessel cord (single artery) (2), placental infarction (2), oligohydramnios (2), placenta was not truly placenta previa but adjacent to (1), small placenta (1), dry placenta (1), infected placenta (1), thickening of placenta (1), bleeding (1), passed presumably placental pieces post-partum (1), marginal cord insertion and possible premature calcium spots on placenta (1), not reported (1)

2 Types of medications: acetaminophen (3); anti-emetic (3) [ondansetron (1), promethazine (1), unspecified(1)]; antibiotics (2); levothyroxine (1); diphenhydramine (1); paroxetine and fluoxetine (1); loratadine (1); acetaminophen-hydrocodone (1); progesterone suppository (1); tightening of uterus (1); hydrocortisone cream (1); not reported (1)

${ }^{3}$ Types of infection: yeast infection (2 patients), urinary tract infection (1), bacterial vaginosis (1), chest infection (1), sinus infection (1), group B streptococcal infection (1)

${ }^{4}$ Types of trauma / accident: car accident (2); fall (1); Twin Towers crashing (1) 